\title{
Preventing central nervous system spread in diffuse large B-cell lymphoma - novel approaches needed
}

Mark Roschewski

\author{
Lymphoid Malignancies Branch, Center for Cancer Research, National Institutes of Health, Bethesda, MD, USA \\ E-mail: MARK ROSCHEWSKI - mark.roschewski@nih.gov
}

doi:10.3324/haematol.2021.278559

(C2021 NIH (National Institutes of Health)

A number of retrospective datasets have addressed the controversial topic of chemotherapy as central nervous system (CNS) prophylaxis during frontline management of diffuse large B-cell lymphoma (DLBCL). ${ }^{1-5}$ Despite the fact that CNS spread is a feared and often terminal complication of DLBCL, there is not a broad consensus regarding which patients should receive CNS prophylaxis or the most effective method of delivery. Overall, the incidence of CNS relapse across all subsets of DLBCL is only about $5 \%$, but some clinical risk factors, including the involvement of specific anatomic sites, are associated with a significantly higher rate of CNS spread. Furthermore, we are beginning to uncover the biological basis for DLBCL involving the CNS as specific genetic subtypes demonstrate an inherently higher rate of CNS tropism. ${ }^{6-8}$ The CNS International Prognostic Index (CNS-IPI) is a commonly used risk model that stratifies patients into risk categories; ${ }^{9}$ combining this model with the cell-of-origin phenotype may improve selection of patients. ${ }^{10}$ However, even the most robust predictive models cannot overcome the fundamental problem that the chemotherapy agents most effective for the cure of systemic DLBCL do not reliably penetrate the blood-brain barrier (Figure 1). ${ }^{11}$ Conversely, methotrexate, which reliably penetrates the CNS, is not highly effective for DLBCL. The most commonly used prophylactic strategy is repeated intrathecal injections of chemotherapy such as methotrexate during frontline therapy, but since brain parenchymal sites are the commonest site of CNS relapse, some advocate the use of deeply penetrant drugs such as high-dose methotrexate. ${ }^{3,12}$ No randomized prospective study has directly addressed this specific issue and, as a result, practice patterns rely on consensus guidelines and vary widely across institutions and individual providers. ${ }^{13}$ In essence, the debate about optimal delivery methods is a "race to the bottom" that compares two strate-
Patient with lymphoma lesions

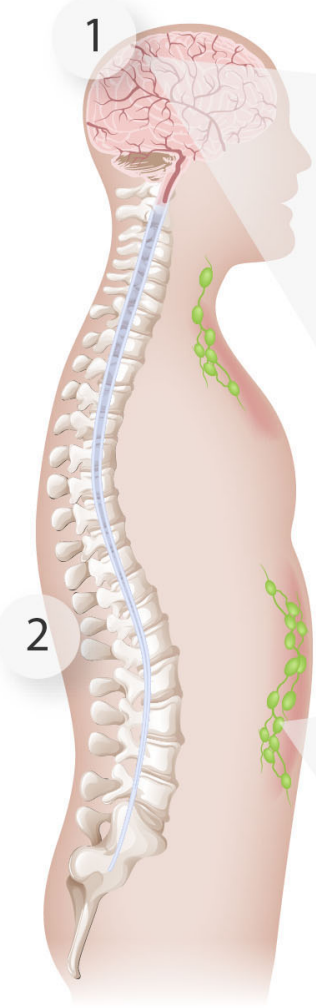

Blood brain barrier

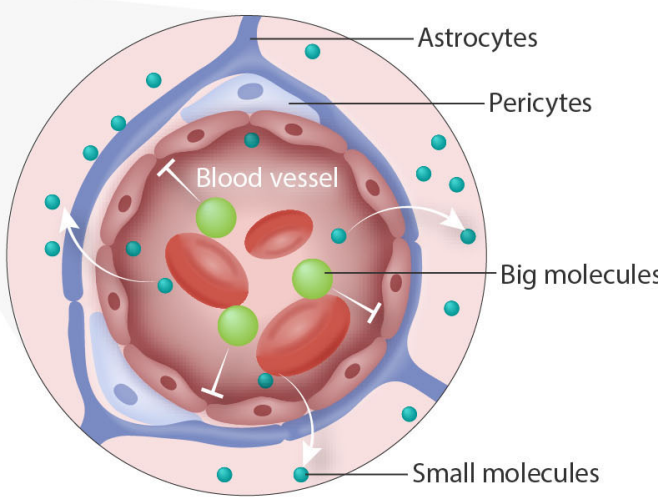

Enlarged lymph nodes

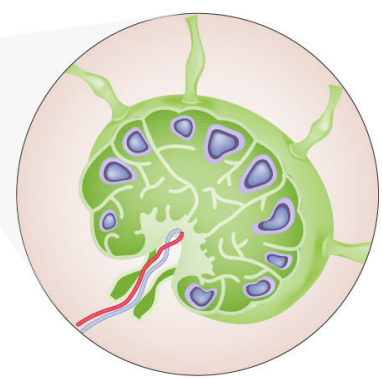

\section{Therapeutic options}

\section{Systemic chemotherapy}

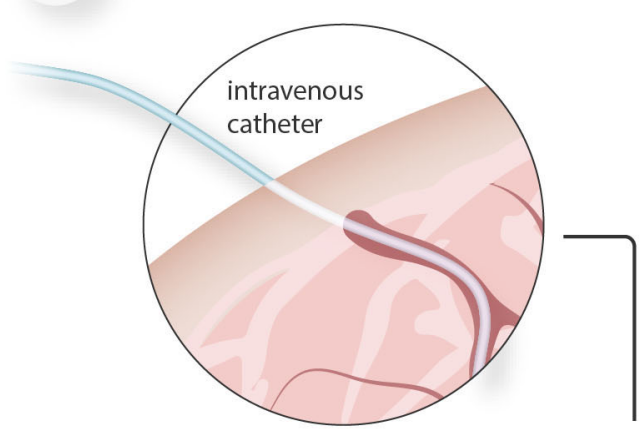

4 Intrathecal chemotherapy

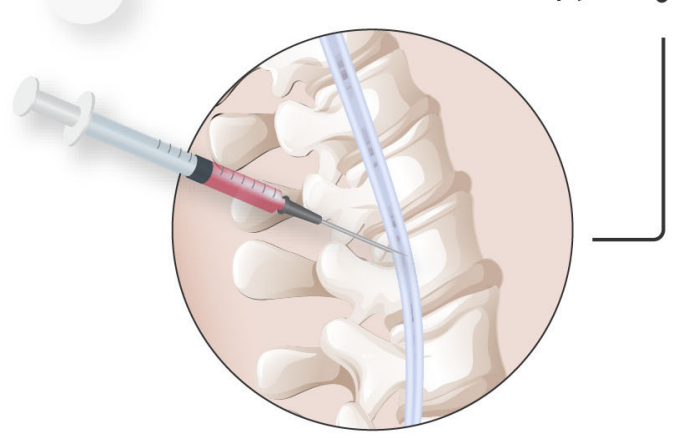

Figure 1. A subset of patients with diffuse large B-cell lymphoma are at high-risk of disease spread to the central nervous system and are often treated with chemotherapy prophylaxis. A critical barrier to effective central nervous system (CNS) prophylaxis is the blood-brain barrier (1) which limits the entry of the chemotherapy agents that are most effective for systemic diffuse large B-cell lymphoma (DLBCL) (2). Current therapeutic options for CNS chemotherapy prophylaxis are systemic chemotherapy (3) or intrathecal chemotherapy (4) which are both limited in efficacy and increase toxicity. Novel small molecule inhibitors that effectively penetrate the blood-brain barrier are being tested in DLBCL involving the CNS and may improve treatment options. 
gies that do not adequately address the clinical problem.

Although retrospective series are effective at generating hypotheses or identifying specific issues that warrant further study, it is nearly impossible to control for all the permutations of approaches to CNS prophylaxis as there is truly no standard approach. The best available data suggest that the most common approach to CNS prophylaxis involves repeated intrathecal injections of methotrexate or cytarabine during frontline therapy, while only a significant minority of patients receive high-dose methotrexate at a median dose of $3.5 \mathrm{~g} / \mathrm{m}^{2}$ either during frontline therapy or immediately following. Notably, patients who receive high-dose methotrexate may also receive concomitant intrathecal chemotherapy which blurs this arbitrary distinction. Furthermore, most datasets demonstrate that nearly half of patients considered high-risk receive no form of CNS prophylaxis. This observation highlights that patient-related factors, such as age and perceived ability to tolerate treatment-related toxicity, greatly influence treatment decisions beyond prognostic scores and/or involvement of extranodal sites. Since all forms of CNS prophylaxis have clinically meaningful toxicities, this underscores the fact that an important limitation of all available datasets is selection bias. Finally, no form of CNS prophylaxis is universally effective and the rate of CNS relapse in patients who receive prophylaxis is typically about $5 \%$ after 2 to 3 years of follow-up. In recognition that CNS relapses may be late events, the actual risk reduction of any form of CNS prophylaxis with chemotherapy is likely modest at best and currently employed strategies may simply delay the timing of CNS recurrence. ${ }^{14}$

The risk of CNS involvement is not equally distributed across all subsets of DLBCL, however, which may allow for precision medicine strategies. In fact, DLBCL is not a single disease but comprises a spectrum of aggressive lymphomas with striking underlying genetic diversity. The current classification system recognizes both activated B-cell (ABC) DLBCL and germinal center B-cell (GCB) DLBCL as distinct molecular subtypes and introduced a new entity, high-grade B-cell lymphoma, defined by the presence of $M Y C$ and $B C L 2$ and/or $B C L 6$ rearrangements (HGBCL-DH/TH) ${ }^{15}$ Indeed, patients with $\mathrm{ABC}$ (nonGCB) DLBCL subtype have an overall higher risk of CNS relapse. ${ }^{10}$ Furthermore, recent multiplatform genomic profiling studies have identified genetic subtypes of DLBCL with shared genetic features. ${ }^{67}$ One genetic subtype, $\mathrm{MCD}$, is characterized by frequent co-occurrence of MYD $88^{1265 P}$ and $C D 79 B$ mutations, prominent immuneediting features, and PIM1 mutations. ${ }^{6}$ These tumors occur almost exclusively within $\mathrm{ABC}$ DLBCL and frequently involve extranodal sites including the testes, breast and CNS. ${ }^{6}$ It is noteworthy that a separate multiplatform genomic profiling study described a very similar subtype termed Cluster 5 (C5) tumors which were characterized by MYD88 $8^{1265 P}$ and $C D 79 B$ mutations, gain of 18q, and PIM1 mutations and also exhibited a propensity for extranodal sites, including the CNS and testes.? Furthermore, a recently reported series of 26 cases of secondary DLBCL of the CNS confirmed a higher prevalence of MCD subtype than that observed in a reference cohort of relapsed DLBCL without CNS spread (38\% vs. $8 \%$,
$P=0.003) \cdot{ }^{16}$ In this study, the majority of other DLBCL cases with CNS spread were either HGBCL-DH/TH or associated with TP53 mutations. Another recent study investigated the genomic predictors of CNS relapse in 82 cases of primary testicular DLBCL which has a strong predilection for CNS spread. ${ }^{17}$ The authors identified $B C L 6$ and/or PDL1 or PDL2 rearrangements as the most common genetic aberrations associated with CNS relapse after treatment for primary testicular DLBCL. Although the precise mechanisms by which various genetic aberrations cooperate to promote CNS spread remain undetermined, these results suggest that a more nuanced understanding of the molecular biology of DLBCL involving the CNS may lead to novel therapeutic targets.

In order to improve clinical outcomes, however, novel therapies with demonstrable efficacy within genetically defined subtypes will be necessary. Multiple clinical studies have reported impressive clinical activity of the Bruton tyrosine kinase (BTK) inhibitor ibrutinib and ibrutinib-based regimens in DLBCL involving the CNS, including patients who are refractory to chemotherapy. ${ }^{18,19}$ Even though a randomized phase III study did not show an overall benefit from adding ibrutinib to R-CHOP as part of frontline therapy for non-GCB DLBCL, certain subsets appeared to have improved outcomes. ${ }^{20}$ Further studies of BTK inhibitors with R-CHOP are currently ongoing and should provide additional data regarding rates of CNS relapse. In addition, the immunomodulatory agent lenalidomide has demonstrated good clinical activity and favorable safety in DLBCL involving the CNS. ${ }^{21}$ Lenalidomide has also been added to R-CHOP as part of frontline therapy for DLBCL which may benefit certain subsets of DLBCL. ${ }^{22}$ The currently available data do not support the use of either ibrutinib or lenalidomide as part of frontline therapy to prevent CNS spread of DLBCL, but all clinical trials testing novel agents should report CNS-specific outcomes within genetically defined subtypes.

In summary, chemotherapy as CNS prophylaxis is not universally effective no matter what the delivery method, and the prevention and treatment of CNS relapse remain unmet clinical needs in the management of DLBCL. Penetration of the blood-brain barrier is an important consideration, but improved therapies will be required to overcome intrinsic chemotherapy resistance. A nuanced mechanistic understanding of targetable pathways underpinning DLBCL involving the CNS has led to novel targeted agents and immunotherapy approaches that demonstrate promising clinical activity and good CNS penetrance. Novel agents that target oncogenic drivers based on the underlying biology of DLBCL subtypes may ultimately prove to be the most effective way to prevent and/or treat CNS recurrence.

\section{Disclosures}

No conflicts of interest to disclose.

\section{References}

1. Puckrin R, El Darsa H, Ghosh S, Peters A, Stewart DA. Lack of effectiveness of intravenous high-dose methotrexate for prevention of CNS relapse in patients with high-risk DLBCL: a retrospective analy- 
sis from Alberta, Canada. Blood. 2020:136(Suppl 1):26-27.

2. Orellana-Noia VM, Reed DR, Sen JM, et al. CNS prophylaxis during front-line therapy in aggressive non-Hodgkin lymphomas: realworld outcomes and practice atterns from 19 US academic nstitutions. Blood. 2020;136(Suppl 1):27-28.

3. Eyre TA, Djebbari F, Kirkwood AA, Collins GP. Efficacy of central nervous system prophylaxis with stand-alone intrathecal chemotherapy in diffuse large B-cell lymphoma patients treated with anthracycline-based chemotherapy in the rituximab era: a systematic review. Haematologica. 2020;105(7):1914-1924.

4. Eyre TA, Kirkwood AA, Wolf J, et al. Stand-alone intrathecal central nervous system (CNS) prophylaxis provide unclear benefit in reducing CNS relapse risk in elderly DLBCL patients treated with R$\mathrm{CHOP}$ and is associated increased infection-related toxicity. Br J Haematol. 2019;187(2):185-194.

5. Cheah CY, Herbert KE, O'Rourke K, et al. A multicentre retrospective comparison of central nervous system prophylaxis strategies among patients with high-risk diffuse large B-cell lymphoma. Br J Cancer. 2014:111(6):1072-1079.

6. Schmitz R, Wright GW, Huang DW, et al. Genetics and pathogenesis of diffuse large B-cell lymphoma. N Engl J Med. 2018;378(15):13961407.

7. Chapuy B, Stewart C, Dunford AJ, et al. Molecular subtypes of diffuse large B cell lymphoma are associated with distinct pathogenic mechanisms and outcomes. Nat Med. 2018;24(5):679-690.

8. Wright GW, Huang DW, Phelan JD, et al. A probabilistic classification tool for genetic subtypes of diffuse large $B$ cell lymphoma with therapeutic implications. Cancer Cell. 2020;37(4):551-568.e14.

9. Schmitz N, Zeynalova S, Nickelsen M, et al. CNS International Prognostic Index: a risk model for CNS relapse in patients with diffuse large B-cell lymphoma treated with R-CHOP. J Clin Oncol. 2016;34(26):3150-3156.

10. Klanova M, Sehn LH, Bence-Bruckler I, et al. Integration of cell of origin into the clinical CNS International Prognostic Index improves CNS relapse prediction in DLBCL. Blood. 2019;133(9):919-926.

11. Arvanitis CD, Ferraro GB, Jain RK. The blood-brain barrier and blood-tumour barrier in brain tumours and metastases. Nat Rev Cancer. 2020;20(1):26-41.

12. Abramson JS, Hellmann M, Barnes JA, et al. Intravenous methotrexate as central nervous system (CNS) prophylaxis is associated with a low risk of CNS recurrence in high-risk patients with diffuse large Bcell lymphoma. Cancer. 2010;116(18):4283-4290.

13. McKay P, Wilson MR, Chaganti S, Smith J, Fox CP, Cwynarski K. British Society of Haematology. The prevention of central nervous system relapse in diffuse large B-cell lymphoma: a British Society for Haematology good practice paper. Br J Haematol. 2020;190(5):708714.

14. Ambady P, Holdhoff M, Bonekamp D, Wong F, Grossman SA. Late relapses in primary CNS lymphoma after complete remissions with high-dose methotrexate monotherapy. CNS Oncol. 2015;4(6):393398.

15. Swerdlow SH, Campo E, Pileri SA, et al. The 2016 revision of the World Health Organization classification of lymphoid neoplasms. Blood. 2016;127(20):2375-2390.

16. Ollila TA, Kurt H, Waroich J, et al. Genomic subtypes may predict the risk of central nervous system recurrence in diffuse large B-cell lymphoma. Blood. 2021;137(8):1120-1124

17. Twa DDW, Lee DG, Tan KL, et al. Genomic predictors of central nervous system relapse in primary testicular diffuse large B-cell lymphoma (DLBCL). Blood. 2021;137(9):1256-1259.

18. Lionakis MS, Dunleavy K, Roschewski M, et al. Inhibition of B cell receptor signaling by ibrutinib in primary CNS lymphoma. Cancer Cell. 2017;31(6):833-843.e5.

19. Grommes C, Pastore A, Palaskas N, et al. Ibrutinib unmasks critical role of Bruton tyrosine kinase in primary CNS lymphoma. Cancer Discov. 2017;7(9):1018-1029

20. Younes A, Sehn LH, Johnson P, et al. Randomized phase III trial of ibrutinib and rituximab plus cyclophosphamide, doxorubicin, vincristine, and prednisone in non-germinal center B-cell diffuse large Bcell lymphoma. J Clin Oncol. 2019;37(15):1285-1295.

21. Ghesquieres $\mathrm{H}$, Chevrier $M$, Laadhari $M$, et al. Lenalidomide in combination with intravenous rituximab (REVRI) in relapsed/refractory primary CNS lymphoma or primary intraocular lymphoma: a multicenter prospective 'proof of concept' phase II study of the French Oculo-Cerebral Lymphoma (LOC) Network and the Lymphoma Study Association (LYSA). Ann Oncol. 2019;30(4):621-628.

22. Nowakowski GS, Hong F, Scott DW, et al. Addition of lenalidomide to R-CHOP improves outcomes in newly diagnosed diffuse large Bcell lymphoma in a randomized phase II US Intergroup Study ECOG-ACRIN E1412. J Clin Oncol. 2021;39(12):1329-1338.

\title{
All in the family: back-to-back kinase inhibitors for the treatment of chronic lymphocytic leukemia
}

\author{
Meghan C. Thompson, Lindsey E. Roeker and Anthony R. Mato \\ Leukemia Service, Memorial Sloan Kettering Cancer Center, New York, NY, USA \\ E-mail: ANTHONY R. MATO - matoa@mskcc.org
}

doi:10.3324/haematol.2021.278535

I this issue of Haematologica, Rogers et al. address a key sequencing question in the management of chronic lymphocytic leukemia (CLL) by reporting the results of the largest prospective clinical trial evaluating acalabrutinib for the treatment of CLL following intolerance to ibrutinib. ${ }^{1}$ While the Bruton tyrosine kinase (BTK) inhibitor ibrutinib has led to a paradigmatic shift in the treatment of CLL away from chemoimmunotherapy, high rates of ibrutinib discontinuation remain a major problem.

Real-world evidence and long-term follow-up from clinical trials of ibrutinib have established that drug intolerance due to toxicity, rather than progressive CLL, is the most common reason for discontinuation of ibrutinib treatment. ${ }^{2-4}$ Real-world data from 616 CLL patients treated with ibrutinib in clinical practice documented that $41 \%$ of patients discontinued ibrutinib (median follow- up 17 months), and more than half of all discontinuations were due to toxicity. ${ }^{2}$ Real-world evidence from the UK documents high rates of ibrutinib discontinuation due to reasons other than disease progression $(17.5 \%){ }^{3}$ Furthermore, similar patterns have emerged with longer follow-up data from clinical trials, with more patients discontinuing ibrutinib due to toxicity than because of CLL progression. At 5 years of follow-up of the RESONATE-2 trial of ibrutinib for initial treatment of CLL, $41 \%$ of patients had discontinued ibrutinib therapy, with a $21 \%$ discontinuation rate due to adverse events including atrial fibrillation. ${ }^{4}$ Furthermore, in a pooled analysis of CLL patients treated with ibrutinib on three randomized phase III studies, $11 \%$ of patients permanently discontinued ibrutinib due to adverse events and $13 \%$ of patients required dose reductions due to adverse events, highlighting the significant impact of adverse events while on 